\title{
On Lin Yutang's Translation Strategies of Geographical Terms in Six Chapters of a Floating Life
}

\author{
Miaojun Lu \\ Longdong University, Qingyang 745000, Gansu, China \\ Email: 18793406469@163.com
}

Abstract: This paper takes Lin Yutang's translation work Six Chapters of a Floating Life as the research object, using the method of combining comparison and case analysis to explore Lin's strategies in the translation of geographical terms. The results show that Lin Yutang adopted foreignization as the major strategy and domestication as the secondary strategy on the whole, and his translation methods are flexible and mature. Lin Yutang's not only successfully explains the culture of Chinese geographical terms, but also provides high reference value for the English translation of geographical terms in other Chinese literary works.

Keywords: Lin Yutang, Six Chapters of a Floating Life, geographical terms, foreignization, domestication

\section{Introduction}

\subsection{Introduction to Six Chapters of a Floating Life}

The Six Chapters of a Floating Life are autobiographical prose written by Shen Fu (1763-1822) of Changzhou and Jiaqing emperor (1808) in Qing Dynasty. The work describes Shen Fu and his wife Chen Yun's deep love for each other. They are indifferent to fame and wealth and want to live an ordinary and artistic life. Due to the oppression of feudal ethics and living in poverty, in the end, the ideal of their life is disillusioned.

\section{The analysis of Lin's translation strategies of geographical terms in Six Chapters of a Floating Life}

\subsection{Lin's translation methods adopted in Six Chapters of a Floating Life}

\subsubsection{Transliteration}

Lin Yutang is one of the first practitioners of transliteration of Chinese geographical terms. However, influenced by the background of the times and international spelling habits, transliteration is widely used in Lin's translation, which is a major feature of his translation.

1. For thirty years I worked as a government clerk in different yamen and practically visited every province expect Szechuen, Kwenichow and Yunnan (Lin Yutang, 2019: 203)

In this case, 蜀中comes from the Annals of Huayang State and Liu XianZhuZhi of Chang Hu of Jin Dynasty: in the 19th year of Jian'an (A.D.214). Then黔中comes from Qianzhong County set up Chu state in Warring States period. 滇is an ancient ethnic name. It is near the Dianchi Lake in the east of Yunnan Province in China. It is named Yunnan because it is to be south of Yunling mountain. Lin Yutang adopted the transliteration method of Wade- System to translate these geographical terms, which were translated as Szechuen, Kwenichow and Yunnan respectively. This kind of translation method belongs to the strategy of foreignization. The advantages of Lin's translation is to greatly improve the accuracy of cross language application of geographical terms.

\subsubsection{Literal translation}

Literal translation is a translation method that keeps both the content and the form of the original text. Literal translation means not only to clarify the meaning of the original completely and accurately, but also to keep the original style without any thoughts of adding or deleting the original at will.

1. His home was situated by the side of the Tower of Mist and Rain [at Kashing], and had an open tower called Moonin-the-Water Lodge overlooking the river. The Tower of Mist and Rain was in the middle of the Mirror Lake, and had an open terrace looking out on green willows on the banks all around (Lin Yutang, 2019: 219)

The geographical term烟雨楼means that the scenery is like a dream in the misty rain, so it is named. The Mirror Lake 
used to be called陶塘. It was a man-made lake in the Southern Song Dynasty. Because of its clear water, it looks like a round mirror, so it is called镜湖. In the case, through the research, it is found that Lin adopted literal translation for the above three geographical terms, this method belongs to the strategy of domestication. The translation can well express the meaning and style of the original text.

\subsubsection{Literal translation + free translation}

1. "The moon is so beautiful to-night," I said. "Let's go up to the Flying Stork Pavilion,"suggested Chuyi (Lin Yutang, 2019: 272)

In 1078, Zhang Tianji of the Northern Song Dynasty built a pavilion in Yunlong mountain. Every morning he released cranes in the pavilion, which was named放鹤亭. In the case, 放鹤as a specific term of geographical terms, Lin adopted literal translation, while亭as a generic term, he adopted free translation. Hence, this translation strategy belongs to the combination of foreignization and domestication. This kind of translation method not only enable us and readers to have deep understanding of the meaning of landscape geographical terms, but also appreciate the simplicity and flexibility of the translation method, and also take into account the surface structure of the translation and the deep meaning of the original.

\subsubsection{Transliteration + free translation}

1. It was summer then and we would get up very early, pass the Ch'ient'ang Gate and the Chaoch'ing Temple. Come down the Broken Bridge and sit down on its stone balustrade (Lin Yutang, 2019: 207)

钱塘门was built in Sui Dynasty and is one of the ten ancient gates in Hangzhou. 昭庆寺 is located on the north bank of West Lake in Hangzhou, it was built in Five Dynasties. As for the origin of the Broken Bridge, there are two reliable theories at present. The first one is that from the Bai Causeway of Autumn Moon and Calm Lake to the bridge deck. It seems to be disconnected, so this bridge is called the broken bridge. The second reliable theory is that Zhang Dai wrote in the West Lake dream at the end of the Ming Dynasty: peach trees and willows were planted along the bank of the Bai Causeway, and the moonlight is shining down, the scattered moonlight was like snow, which means "Remnant Snow on the Broken Bridge in Winter.” In this case, for the specific terms of geographical terms such as “钱塘” “昭庆” “断” Lin adopted the method of transliteration; the generic terms such as “门” “寺” “桥” are translated by free translation. So, “钱塘门” “昭庆寺” “断桥” are translated respectively as Ch'ient'ang Gate, Chaoch'ing Temple and Broken Bridge. These translation methods belong to the strategy of foreignization. The advantage of such translation is to retain the information of the source text culture.

\subsubsection{Free translation}

Free translation means that both the specific and generic terms of geographical terms are translated by free translation, which shows that in some cases, literal translation of geographical terms is not feasible.

1. At this time, my younger brother Ch'it'ang was going to get married, and was moved to Ts'angmi Ally by the Bridge of Drinking Horses (Lin Yutang, 2019: 07)

According to Records of the Grand Historian, the name of 饮马桥comes from Wang Jian Visit to PingJiang Mansion. In the above case, Lin adopted the free translation method, which belongs to the strategy of foreignization. This kind of translation strategy keeps the differences of national culture well.

\subsubsection{Footnotes}

The situation supplemented by footnotes refers to that some geographical terms still do not express clearly the meaning of geography, culture, history and so on after using the above translation methods. At this time, it needs to be supplemented by footnotes at the end of the page.

1. There was a temple on top in honour of a certain General Mei, I do not know of what dynasty (Lin Yutang, 2019: 245)

Footnote: This was General Mei Chuan who was one of the first Chinese colonizers of Kwangtung at the beginning of the Han Dynasty

Example 1 introduces famous General Mei Chuan Han Dynasty of China.Lin made a detailed footnote to explain the origin of Mei General's Temple to westerners. As is known to all, Mei General's Temple is a place names of allusions, the translation of footnotes helps readers to know the culture of geographical terms in China.

In Lin's translation, there are different ways to translate the same type of geographical terms. I choose some translation cases and make a brief analysis. For example靖海门is translated as “Tsinghai gate”, but幽兰门is translated as “yulanmen”. The reason is that幽兰门has water sinuses to go out and "there was a water gate". 吼山is translated as Hush-an Hill, while 邓尉山and灵岩山are translated as “Teng'wei Hill” and “Lingyen hill” respectively. The reason is that the part of the specific term in 吼山is monosyllabic. Therefore, both the part of the specific term and the part of the generic term are transliterated and linked, and then the part of the generic term literal translation is repeated. For example, 华山is translated as Huashan Hill, but the reasons for this translation are not the same. For example, 锡山and 虞山are translated as Hsishan and Yushan Hill respectively, because锡山 only refers to a geographical term, while虞山refers to the mountain of scenic spots. In addition, 
小孤山 was translated into "Little Orphan". In the original text, the literal translation is enclosed with quotation marks, so its meaning perfectly clear. Because 小孤山calls this name because "one peak in the center of the river rising, with no other peaks around it”, so the metaphorical effect of 小孤山is well preserved.

\section{Conclusion}

Through literature research, observation and case study of a large number of examples of geographical terms, it is found that Lin's translation of geographical terms in Six Chapters of a Floating Life has the following characteristics.

In general, geographical terms are divided into the following four main types:place names, landscape names, famous building names and place names of allusions. Moreover, place names consist of two parts: specific terms and generic terms. Specific term indicates the general geographical entities with obvious commonness, and it is a specific name that distinguishes different individuals in a common geographical entity. Generic term refers to the type of geographical terms.

Besides, by citing a large number of English translation of examples of geographical terms, it is found that Lin mainly adopted direct transliteration, literal translation, transliteration plus free translation, free translation, literal translation plus free translation, footnote. In sum, Lin's translation methods are flexible and varied for the complicated geographical terms.

What's more, through observation and analysis of a large number of geographical translation, the result of the analysis indicates foreignization strategy predominates over the domestication strategy in translating the geographical terms.

In summary, the study of the translation strategies of geographical terms will contribute to the development of China's foreign tourism industry and better convey the connotation of geographical terms in other literary works.

\section{References}

[1] Nida, Eugene. A. The Theory and Practice of Translation. Shanghai: Foreign Language Education, 2006.

[2] Lin, Yutang. The Wisdom of China. Beijing: Foreign and Teaching and Research Press, 2009.

[3] Munday, Jeremy. Introducing Translation Studies: Theories and Applications. London: Routledge, 2001.

[4] Chen Aiming. (2006) A Study on the Translation Strategies of English Translation of Six Chapters of a Floating Life. Capital Normal University, Beijing.

[5] Chen Wei. The translation style of Lin Yutang's Six Chapters of a Floating Life. Anhui Literature. 2009; (08).

[6] Lin Wei. Translator's Subjectivity Demonstrated in Lin Yutang's Translation of Six Chapters of a Floating Life. Journal of Fujian Commercial College. 2016; (3): 76-80.

[7] Shen Fu. Translated by Lin Yutang and Li Hui. Six Chapters of a Floating Life. Changsha: Hunan Literature and Art Publishing House; 2019.

[8] Wang Baorong. (2016) Strategies for place names in Six Chapters of a Floating Life. Zhejiang University of Finance and Economics, Hangzhou.

[9] Zhu Jiachun. (2019) An Empirical Study and Cross-Cultural Interpretation of the Cultural-specific Translation Strategies of Lin Yutang's English Translation of Six Chapters of a Floating Life. Tsinghua University, Beijing. 